\title{
Recurrent Anal Mucinous Adenocarcinoma
}

National Cancer Institute

\section{Source}

National Cancer Institute. Recurrent Anal Mucinous Adenocarcinoma. NCI Thesaurus.

Code C153576.

The reemergence of anal mucinous adenocarcinoma after a period of remission. 